EVS25

Shenzhen, China, Nov 5-9, 2010

\title{
A Novel Hybrid Control Strategy for Maximising Regenerative Braking Capability In a Battery-Supercapacitor Energy Storage System
}

\author{
Dr Ralph Clague ${ }^{1}$, Dr Ilja Siera, Dr Michael Lamperth \\ ${ }^{1}$ EVO Electric Ltd, Unit 14 Woking Business Park, Woking. E-mail: r.clague@imperial.ac.uk
}

\begin{abstract}
EVO Electric has designed, built and tested the DuoDrive hybrid system based on proprietary axial flux motor technology, and installed it in a London Taxi Cab. The DuoDrive switchable series/parallel hybrid system has demonstrated a $60 \%$ improvement in fuel economy compared to a conventional taxi when operated over an urban drive cycle. As with many hybrid vehicles, a large part of this improvement is attributed to effective and efficient recapture of braking energy. The amount of braking energy that can be recovered, and the efficiency with which it can be returned to the road will therefore have a significant impact on the overall fuel economy of the vehicle. One factor that limits the amount of energy that can be recovered is the allowable charge rate of the battery, as braking events are usually high power and in a hybrid vehicle the battery size is generally small. The vehicle described in this paper has an energy storage system comprised of high power ultra-capacitors and a high energy lithium ion battery connected through a DC/DC converter. This allows efficient, high power transfer under regenerative braking and acceleration, and similarly efficient energy storage over longer timescales. Managing the power flow through the DC/DC converter and therefore the ultra-capacitor voltage, is a key control parameter that affects the efficiency of the overall system. This paper presents the energy storage system layout and demonstrates how different DC/DC converter control strategies can affect the system energy efficiency.
\end{abstract}

Keywords - Hybrid, electric, vehicle, efficiency, control

\section{Introduction}

Hybridising a conventional internal combustion engine by adding an electric motor is a proven method of improving fuel economy of vehicles driven over an urban i.e. stop start drive cycle [1]. There are a number of different ways of implementing a hybrid drivetrain but they all share common goals: the prime objective is to recapture kinetic energy from braking events that

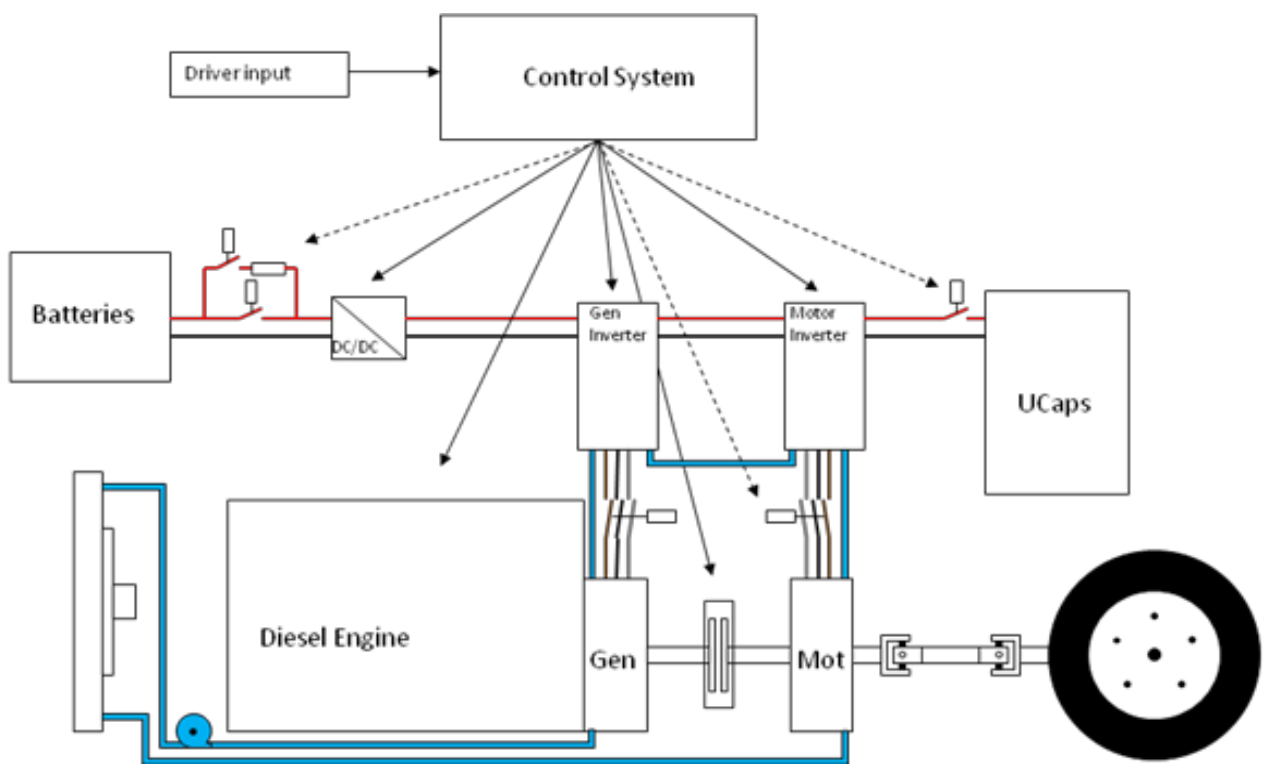

Figure 1 - DuoDrive Hybrid System layout 
would otherwise be dissipated as heat, the second objective is to minimize the time the internal combustion engine spends at light loads or otherwise off the maximum efficiency design point. The majority of the benefit of having a hybrid powertrain comes from the former, so maximizing the efficiency with which energy is captured, stored and returned to the road is of key importance to maximizing the fuel economy of the vehicle.

The DuoDrive hybrid system for passenger and light commercial vehicles has been developed at EVO Electric Ltd (EVO) based on EVO axial flux, permanent magnet synchronous motor and generator technology. DuoDrive offers three modes of vehicle operation: pure electric, parallel hybrid and series hybrid. DuoDrive hardware has been installed in a London Taxi with a proprietary control system designed, programmed and tested at EVO Electric, as a validation of their axial flux electric machines and hybrid system design capability, and demonstration of the potential for fuel economy improvements of a plug-in hybrid taxi.

The basic system architecture shown in figure 1 has a novel feature in that the energy storage system is comprised of a lithium ion battery pack and an ultracapacitor array. This is in order to minimise component sizes and preserve the life of the battery. In the taxi installation the gearbox is replaced with a single unit comprising the generator, clutch and electric motor.

In this application in the London Taxi, the hybrid system is comprised of the following hardware:

- Engine - Ford Duratorq (Puma) 2499cc Diesel

- Generator - EVO Electric AFG-140

- Generator Inverter - EVO Electric BEK115

- Motor - EVO Electric AFM-140

- Motor Inverter - EVO Electric BEK115

- Batteries - LifeBATT 10815 module (3x)

- Ultracapacitors - Maxwell BCAP 350 (300x)

- DC/DC Converter - 9kW nominal bidirectional buck-boost

- Control System - Beckhoff PC and Ethercat system, control software developed in Simulink

The drive motor is connected to the rear wheels through a differential with a reduction ratio of $4.1: 1$, the rear wheel rolling radius is $0.325 \mathrm{~m}$.
The DuoDrive system offers reduced complexity compared to other OEM dual mode hybrids [2], increased efficiency and reduced implementation cost due to the minimization of energy storage and transfer component sizes and the gearbox being eliminated.

Arguably the most important part of the system is the hybrid control software. This has been developed at EVO Electric using Matlab-Simulink software and is a rules rather than cost-function based system designed around the following requirements; minimising the number of engineon events in urban driving; operating the engine as close as possible to the peak efficiency point when it is on; maximising the amount of vehicle kinetic energy that can be recovered; minimizing the transfer of power in both directions between the batteries and ultracapacitors.

\subsection{Engine and Generator}

The internal combustion engine used is the original TX2 unit, a Ford Duratorq 2499cc TurboDiesel. The peak efficiency of this engine is at maximum torque and between 1400 and $1500 \mathrm{rpm}$. It can be seen from the combined efficiency map of the diesel engine and AFG-140 is shown in figure 2, that the most efficient region to operate the gen-set in is between 1250 and $1750 \mathrm{rpm}$, depending on the power requirement. In order to optimize the NVH characteristics of the engine, it was run at the lowest speed possible to meet the power requirement.

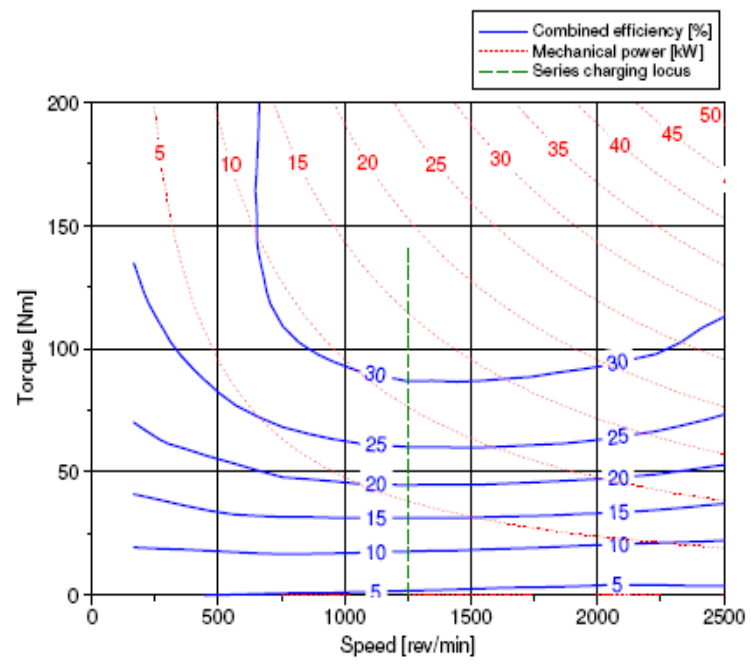

Figure 2 - Combined efficiency map of Diesel engine and EVO AFG-140-4 


\section{System Description}

\subsection{Modes of Operation}

The DuoDrive system allows three modes of operation: pure electric vehicle (EV), series hybrid $(\mathrm{SH})$ and parallel hybrid $(\mathrm{PH})$. Both hybrid modes are on average charge sustaining. This allows the vehicle to operate optimally in the different driving environments of; short urban journeys; extended urban driving; highway cruising. When the system is started up the default mode is EV until the diesel engine is triggered to start, moving the system into $\mathrm{SH}$ mode. There are a number of engine-on triggers, but principally they are: low battery state of charge (SOC) or high sustained power demand that drains the ultracapacitors to battery voltage. Once the engine is on it is run at the highest load and lowest speed possible, in that order of preference, until the batteries regain $80 \%$ SOC, at which point it will turn off. PH mode is engaged at highway speeds and whilst important, is not the main focus of this paper.

\subsection{On-Board Power Flow}

The overall power flow into and out of the energy storage system is shown in figure 3:

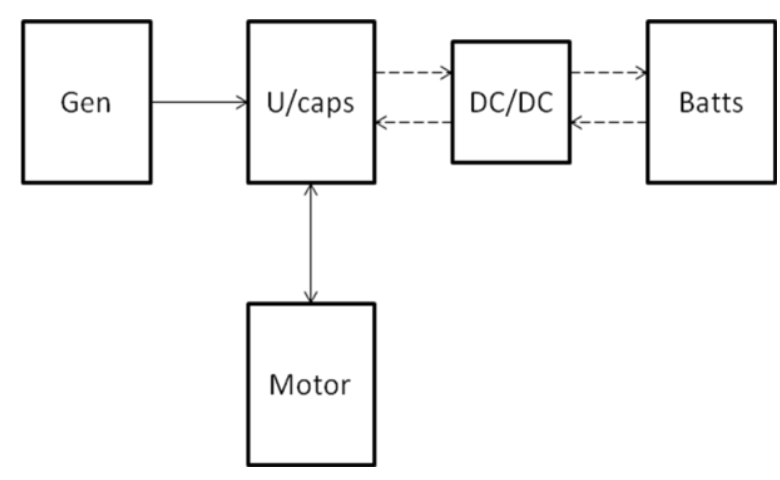

Figure 3 - Power flow in the DuoDrive system

Each vehicle mode (EV, PH, SH) demands a different DC/DC control strategy, but the requirements of the DC Bus voltage remain the same: a DC Bus target voltage is determined and the difference between this and the actual DC Bus voltage is used to determine the DC/DC current setpoint i.e. a simple P-controller. When the actual DC bus voltage rises above the target the batteries are charged from the ultra-capacitors. When the DC bus drops below the target the batteries are discharged into the ultra-capacitors.

\subsection{Bus Target Voltage Calculation}

The DuoDrive hybrid system allows the DC Bus voltage (defined by the state of charge of the ultra-capacitors) to float between battery voltage and a maximum potential of $700 \mathrm{~V}$. The DC/DC converter was sized at $9 \mathrm{~kW}$ nominal as this is the power requirement for driving the vehicle at $30 \mathrm{mph}$ on a flat road. The main method of managing the DC Bus voltage is by controlling the DC/DC link between the ultracapacitors and batteries. There are effectively three conflicting requirements for the ideal DC Bus voltage:

- There must be enough energy available to allow the vehicle to accelerate when required.

- The DC Bus voltage must be larger than the back emf of the electric motor at a given speed, to allow the vehicle to accelerate further up to a maximum speed.

- The ultracapacitors must have sufficient spare capacity to recapture the energy from braking events in urban driving

The first requirement simply implies that the maximum energy possible should be stored in the ultracapacitors, while satisfying the other requirements.

The second requirement derives from the fact that in permanent magnet machines controlled by inverters that do not employ vector control, the back emf $V_{\text {emf }}$, necessarily rises as machine rotational speed increases according to equation (1) where $\mathrm{K}_{\mathrm{e}}$ is the voltage constant in $\mathrm{V} / \mathrm{rad} / \mathrm{s}$ and $\omega$ is the machine rotational speed in $\mathrm{rad} / \mathrm{s}$.

$$
V_{\text {emf }}=K_{e} \omega
$$

In order to increase the speed of the machine, the applied voltage must be greater than the back emf in order to make current flow in the windings. The EVO AFM-140 is produced with three standard winding configurations: 3,4 and 6 pole pairs. The variation of $\mathrm{K}_{\mathrm{e}}$ with the number of machine pole pairs is given in table 1, the torque constant $K_{t}$, is also given for reference. The first requirement of the DC Bus is therefore that the minimum DC Bus voltage shall rise as vehicle speed increases. Table 2 presents the generated back emf for a given vehicle speed. 
Table 1 - Torque and speed constants for standard configurations of EVO AFM-140-4

\begin{tabular}{|l|l|l|}
\hline \multirow{2}{*}{ Motor Type } & \multicolumn{2}{|l|}{ Motor Constants } \\
\cline { 2 - 3 } & $\mathrm{Kt}(\mathrm{Nm} / \mathrm{Amp})$ & $\mathrm{Ke}(\mathrm{V} / \mathrm{rad} / \mathrm{s})$ \\
\hline AFM-140-3 & 1.36 & 0.81 \\
\hline AFM-140-4 & 1.81 & 1.08 \\
\hline AFM-140-6 & 2.72 & 1.62 \\
\hline
\end{tabular}

Table 2 - Induced back EMF of AFM-140-4 at various vehicle speeds

\begin{tabular}{|l|l|l|}
\hline $\begin{array}{l}\text { Vehicle Speed } \\
(\mathrm{km} / \mathrm{h})\end{array}$ & Electric Motor \\
\cline { 2 - 3 } & Speed $(\mathrm{rad} / \mathrm{s})$ & Back EMF $(\mathrm{V})$ \\
\hline 30 & 106 & 192 \\
\hline 50 & 177 & 320 \\
\hline 70 & 247 & 448 \\
\hline
\end{tabular}

The third requirement for the DC Bus is that in order to recover the maximum amount of vehicle kinetic energy possible, there must be sufficient spare capacity in the supercapacitors at the start of a braking event. If all of the difference in vehicle kinetic energy when decelerating from speed $U_{1}$ to speed $U_{2}(\mathrm{~m} / \mathrm{s})$ is stored in the ultracapacitors, this will raise the voltage of the ultracapacitors from $\mathrm{V}_{1}$ to $\mathrm{V}_{2}$. Taking an energy balance with $\mathrm{U}_{2}=0$ and $\mathrm{V}_{2}=700 \mathrm{~V}$, the upper limit of ultracapacitor string voltage, allows calculation of the DC Bus voltage target for a given vehicle speed in order to recapture all of the braking energy. Assuming the vehicle kinetic energy to be $1 / 2 \mathrm{mU}^{2}$ and the energy in the ultracapacitors to be $1 / 2 \mathrm{CV}^{2}$ an energy balance yields equation (2)

$$
V_{1}=\sqrt{V_{2}^{2}-\frac{m}{C} U^{2}}=\sqrt{V_{2}^{2}-2 \frac{E_{K E}}{C}}
$$

Where $\mathrm{C}$ is the capacitance in Farads, and $\mathrm{V}$ is the voltage. However, during real braking events it is not possible to recapture all of the vehicle kinetic energy. The real amount of energy available depends on the vehicle dynamics and losses. An algorithm was developed to calculate the real amount of regenerative energy, $\mathrm{E}_{\mathrm{RG}}$, available such that (3)

$$
V_{1}=\sqrt{V_{2}^{2}-2 \frac{E_{R G}}{C}}
$$

The key conclusion from this part of the analysis is that the DC Bus voltage should drop at higher speeds to allow recapture of all braking energy at high power while keeping it above the increasing back emf. Managing these two different DC Bus voltage requirements is critical to maximizing the efficiency of the hybrid system as a whole.

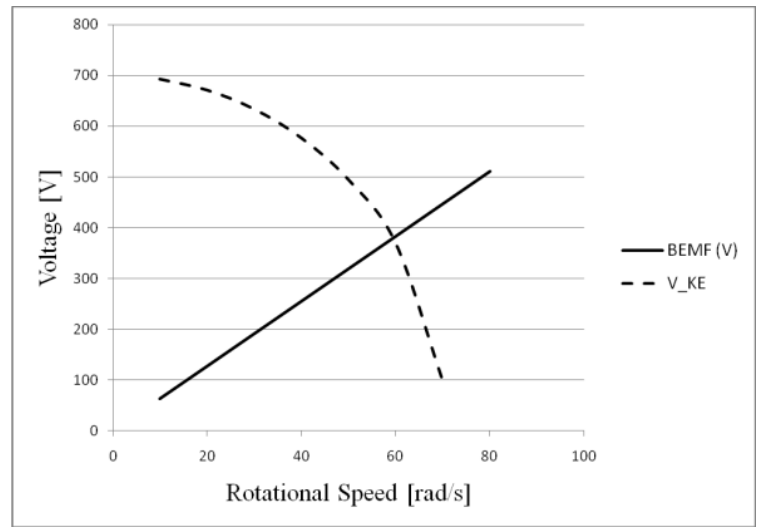

Figure 3 - DC Bus target voltage requirements

Balancing the two major conflicting DC Bus control requirements was achieved by calculating the two DC bus voltage 'setpoints' within the control system, then taking the maximum of the two as the DC Bus voltage target, which the control system then tries to achieve by modulating power flow through the DC/DC converter. Figure 3 shows the changing DC bus requirements with increasing vehicle speed.

\section{Results and Discussion}

Figures 4 and 5 compare the power flow between the battery and supercapacitors for two different control systems; Case1 - the DC Bus target voltage is fixed at $700 \mathrm{~V}$. The control strategy for the DC/DC converter is that, when the DC Bus voltage drops below a given value the current set point ramps up to maximum, transferring energy from the battery to the ultracapacitors. During regeneration of the braking energy, when the ultracapacitor voltage rises above a certain value, the $\mathrm{DC} / \mathrm{DC}$ converter transfers energy back to the batteries; Case 2 - The DC Bus target voltage is dependent on vehicle speed. As with case 1, when the DC Bus voltage drops below a given value the $\mathrm{DC} / \mathrm{DC}$ converter transfers energy from the batteries to the ultracapacitors, and conversely when the ultracapacitor voltage is higher than the target voltage energy is transferred back to the batteries. In this case the control system will attempt to achieve a DC bus voltage target that is vehicle speed dependent. 


\subsection{Case 1}

For Case1 as the vehicle accelerates from 0 to 40 $\mathrm{km} / \mathrm{h}$ the energy in the ultracapacitors drops as they are discharged. The battery then provides as much power as it can in order to acheive the DC Bus target voltage. In phase 2 the vehicle speed is maintained at $40 \mathrm{~km} / \mathrm{h}$, at which point nearly all of the rated $\mathrm{DC} / \mathrm{DC}$ converter power is required to overcome drag and other losses, so the energy in the ultracapacitors rises very slowly. In phase 3 the vehicle speed is reduced from $40 \mathrm{~km} / \mathrm{h}$ to 0 and the vehicle kinetic energy is recaptured through the electric drive motor. The DC/DC swaps to battery charging when the DC Bus voltage target is achieved, which results in the energy that has just been removed from the battery being returned, with all the associated losses. Moreover, due to the limited rate of charge, not all the braking energy available can be stored into the batteries. That additional energy will have to be dissipated by the traditional brakes.

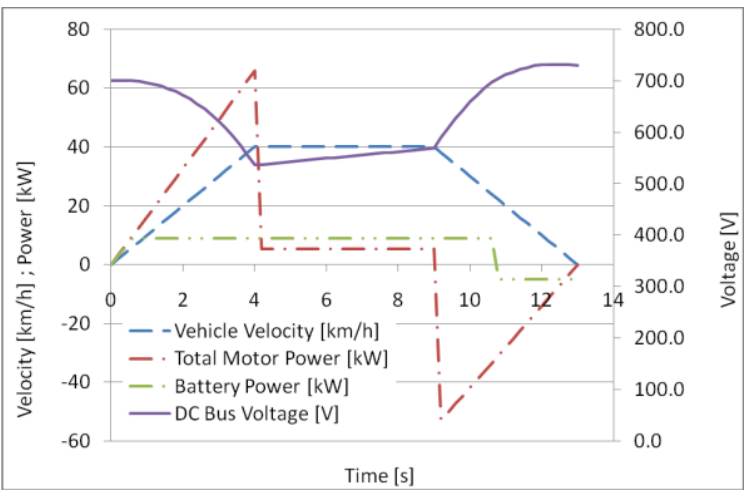

Figure 4 - Vehicle speed, ultracapacitor energy, battery power and motor power for Case 1

If the average efficiencies of the DC/DC converter, ultra-capacitors and batteries are assumed to be $90 \%, 98 \%$ and $92 \%$ respectively the total energy loss due to power transfer through these devices is approximately $23.6 \mathrm{KJ}$.

\subsection{Case 2}

For case 2 the vehicle follows the same speed pattern but this time the target DC bus voltage drops with increasing vehicle speed according to the developed algorithm so that all of the vehicle kinetic energy can be recaptured and stored in the ultracapacitors. The key point to note is that in the braking phase the battery does not charge, it is merely used to help achieve the target DC Bus voltage, which rises as the vehicle slows. Therefore all of the braking energy is recaptured in the ultracapacitors, making full use of them and minimizing the required capacity of the bank and allowing high energy, rather than high power cells to be used in the battery pack. As for case 1, if the average efficiencies of the DC/DC converter, ultra-capacitors and batteries are assumed to be $90 \%, 98 \%$ and $92 \%$ respectively the total energy loss due to power transfer through these devices is approximately $14.8 \mathrm{KJ}$.

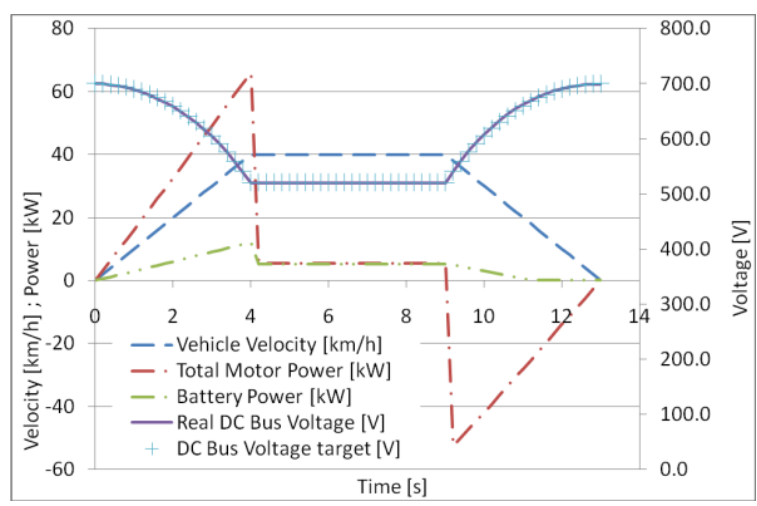

Figure 5 - Vehicle speed, ultracapacitor energy, battery power and motor power for Case 2

The losses in the energy storage system are approximately $60 \%$ higher for case 1 than for case 2.

\section{Conclusion}

EVO Electric have designed, built and tested the DuoDrive hybrid system using a London Taxi as a vehicle platform and demonstrating 60\% improvement in fuel economy in urban driving. A novel and important part of the DuoDrive system is the use of batteries and ultracapacitors, linked with a DC/DC converter as the energy storage system. Correctly determining the ultracapacitor target voltage is a key system design parameter for a system of this type. The novel rules-based strategy developed at EVO Electric allows minimization of the losses in the energy storage system and also, by making full use of the features of each type of storage media, the size of the ultra-capacitor bank and DC/DC converter for the required amount of regenerative energy recapture in urban driving. At extra-urban speeds the minimized size of the ultracapacitor element of the energy storage system, and characteristics of the permanent magnet synchronous motor limits the recoverable kinetic energy such that some must be discarded. The system is therefore optimized for the drive cycle for which the vehicle was intended i.e. up to $30 \mathrm{mph}$ stop-start urban driving. 


\section{References}

[1] Kromer M, Heywood J, Electric Powertrains: Opportunities and Challenges in the US Light Duty Vehicle Fleet LFEE 2007-03 RP. Sloan Automotive Laboratory, Massachusetts Institute of Technology, Cambridge, MA

[2] M Duoba, H Ng, R Larsen - Characterization and comparison of two hybrid electric vehicles (HEVs)Honda Insight and Toyota Prius, SAE TRANSACTIONS, 2001

\section{Authors}

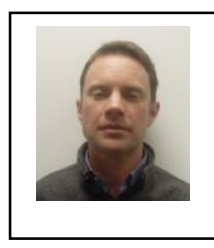

Dr Ralph Clague

EVO Electric, Unit 14 Woking Business Park, Woking, Surrey, UK, GU21 5JY

URL: www.evo-electric.com Tel: +44 7767823465

Email: r.clague@imperial.ac.uk

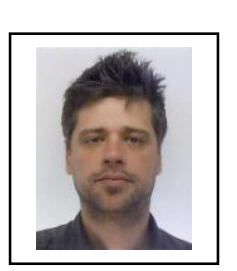

\section{Dr Ilja Siera}

EVO Electric, Unit 14 Woking Business Park, Woking, Surrey, UK, GU21 5JY

Tel: +44 1483745010

Email: ilja.siera@evo-electric.com URL: www.evo-electric.com

\section{Dr Michael Lamperth}

EVO Electric, Unit 14 Woking

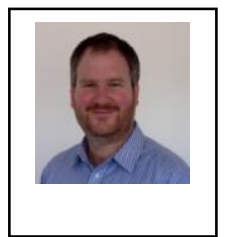

Business Park, Woking, Surrey, UK, GU21 5JY

Tel: +44 1483745010

Email: Michael.lamperth@evoelectric.com

URL: www.evo-electric.com 